\title{
Tapentadol vs. pregabalina asociada a otros opioides en dolor crónico. Análisis de coste-efectividad
}

\author{
M. Avellanal ${ }^{1,2}$, G. Díaz-Reganon ${ }^{1,2}$, A. Orts ${ }^{1,2}$ y S. Soto ${ }^{2,3}$ \\ ${ }^{1}$ Unidad del Dolor. Hospital Universitario de Madrid y Hospital Sanitas La Moraleja. Madrid. \\ ${ }^{2}$ Consultores en Dolor. ${ }^{3}$ Hospital Universitario de Getafe. Madrid
}

Avellanal M, Diaz-Reganon G, Orts A, Soto S. Tapentadol vs. pregabalina asociada a otros opioides en dolor crónico. Análisis de coste-efectividad. Rev Soc Esp Dolor 2014; 21(2): 84-88.

\begin{abstract}
Background: Tapentadol is a new oral analgesic with a dual mode of action as a mu-opioid receptor agonist and as norepinephrine reuptake inhibitor. The cost of treatment can be argued as a problem for prescribing it.

Objective: The aim of this study was to compare the cost-effectiveness of tapentadol to that of associations of other opioids with pregabalin.

Patients and methods: 21 patients suffering chronic pain under treatment with opioids and pregabalin for more than 3 months and pain poorly controlled (VAS > 4) were proposed to change to tapentadol in a progressive manner: First changing the opioid to tapentadol and then removing pregabalin. Pain(VAS), dailycostoftreatmentsandadverseeffectsincidencewere registered.

Results: Four patients returned to prior treatment because of higher incidence of adverse effects. In the group of $17 \mathrm{pa}-$ tients who completed the change of treatment pain improved from 5.7 (VAS) to 3.4 (VAS) ( $<<0.001$ ), and the cost of treatment from $4.57 € /$ day to $3.78 € /$ day $(p<0.05)$.

Conclusion: Tapentadol treatment may be cost-effective when compared with associations of other opioids and pregabalin in patients suffering moderate to severe chronic pain. More extensive studies must be done in this sense.
\end{abstract}

Key words: Chronic pain. Tapentadol. Pregabalin. Opioids. Cost-effectiveness.

Recibido: 16-03-13.

Aceptado: 18-06-13.

\section{RESUMEN}

Fundamento: el tapentadol es un nuevo analgésico con mecanismo de acción dual como agonista opioide $\mu$ e inhibidor de la recaptación de noradrenalina. El coste del tratamiento puede suponer un problema a la hora de prescribirlo.

Objetivo: analizar si el tratamiento con tapentadol puede resultar coste-efectivo frente a otros opioides asociados a pregabalina.

Pacientes y método: se incluyeron 21 pacientes en tratamiento por dolor crónico con opioides asociados a pregabalina y mal control analgésico (EVA > 4). Se les propuso rotar a tratamiento con tapentadol en dos fases: primero sustituyendo el opioide por tapentadol y posteriormente retirando progresivamente la pregabalina. Se registraron el dolor (EVA), el coste diario del tratamiento y la incidencia de efectos adversos antes y tras la introducción del nuevo tratamiento.

Resultados: cuatro pacientes abandonaron el tratamiento y volvieron al previo por mayor incidencia de efectos secundarios. En el grupo restante el dolor mejoró de 5,7 (EVA) a 3,4 (EVA) ( $p<0,001)$, mientras que el coste de tratamiento pasó de $4,57 € /$ día a $3,78 € /$ día $(p<0,05)$.

Conclusión: el tratamiento con tapentadol puede resultar coste-efectivo frente a la combinación de otros opioides con pregabalina en pacientes con dolor crónico moderado-grave. Se requieren estudios más amplios que confirmen estos hallazgos.

Palabras clave: Dolor crónico. Tapentadol. Pregabalina. Opioides. Coste-beneficio.

\section{INTRODUCCIÓN}

El tapentadol, disponible desde agosto de 2011 en España, constituye el primer representante de un nuevo grupo de fármacos analgésicos con un mecanismo de acción 
dual: los agonistas de receptores opioides $\mu$-inhibidores de recaptación de noradrenalina (MOR-NRI) (1). El fármaco actúa como agonista opioide $\mu \mathrm{y}$, al mismo tiempo, como inhibidor de la recaptación de noradrenalina (NRI) en un rango de dosis de 0,1-0,5 microM (2). Se trata de un analgésico opioide que ha demostrado baja afinidad por el receptor $\mu$, hasta 50 veces inferior a la morfina. A pesar de ello su eficacia analgésica es únicamente 2,5 veces inferior, lo que se explica por su mecanismo noradrenérgico asociado (3).

El antagonismo del receptor opioide $\mu$ conduce a una inhibición de la transmisión del dolor en el ámbito de las vías nociceptivas ascendentes mientras que, simultáneamente, se amplifican las vías inhibitorias descendentes del dolor desde la sustancia gris periacueductal hasta el asta posterior de la médula gracias a la inhibición de la recaptación de noradrenalina (4).

Desde el punto de vista farmacocinético presenta un perfil muy atractivo frente a otros opioides: no es un profármaco (como codeína, por ejemplo) y por tanto no tiene que ser activado previo paso por el hígado u otras vías metabólicas; su metabolismo hepático es prácticamente nulo (no llega al $15 \%$ la fracción metabolizada vía cit $\mathrm{P} 450$, por lo que no está sujeto a interacciones con otros fármacos inductores o bloqueadores del mismo como antidepresivos, antiepilépticos, macrólidos, etc.); su unión a proteínas plasmáticas es escasa (no llega al $20 \%$ ); no tiene metabolitos activos ni tóxicos (el $97 \%$ de la dosis administrada se elimina en forma inactiva; su eliminación tiene lugar vía reacciones fase II tipo glucurono y sulfoconjugación, y la consiguiente eliminación renal). La excreción fecal no llega al $1 \%$. No precisa tener en cuenta consideraciones farmacogenómicas que puedan afectar a metabolizadores ultrarrápidos o metabolizadores lentos, como puede ocurrir con otros opioides con marcado metabolismo hepático. Tampoco precisa ajuste de dosis en pacientes con insuficiencia renal leve o moderada (5). Varios estudios clínicos muestran una incidencia de efectos secundarios menor que con otros opioides $(15 \%$ vs. $30 \%)$ con la excepción de la sequedad de boca, que es mayor, y una tasa mucho menor de abandono de tratamiento (6), todo ello en posible relación con su escasa potencia como agonista $\mu$ que parece compensar clínicamente con el mecanismo de acción dual descrito.

En definitiva, se trata de un opioide con novedosa acción mixta agonista $\mu$-NRI con un perfil farmacocinético muy favorable pensando en pacientes de edad, polimedicados y con posibles disfunciones renales o hepáticas asociadas, clínicamente mejor tolerado y que abre definitivamente la puerta al empleo de opioides también en el dolor neuropático.

En España se ha comercializado la presentación retardada en administración de $12 \mathrm{~h}$ con dosis de 25, 50, 100, 150, 200 y $250 \mathrm{mg}$. El coste por día de tratamiento oscila entre $1,47 € /$ día con la posología de $50 \mathrm{mg} / 12$ h y $5,64 € /$ día con la posología mayor de $250 \mathrm{mg} / 12 \mathrm{~h}$.
En comparación con otros opioides, el coste por día de tratamiento es sensiblemente más elevado que con tramadol, morfina, fentanilo transdérmico y buprenorfina transdérmica a dosis equivalentes. Respecto a oxicodona, el coste por día a dosis bajas saldría favorable a este (la equivalente a $50 \mathrm{mg} / 12$ de tapentadol retardado sería oxicodona $10 \mathrm{mg} / 12 \mathrm{~h}$ a $1,10 € /$ día), aunque a dosis altas el balance se inclina claramente a favor del tapentadol (oxicodona $90 \mathrm{mg} / 12 \mathrm{~h}$ suponen $8,36 € /$ día). Algo parecido ocurre comparándolo con hidromorfona. Hay que tener en cuenta que el número de comprimidos por envase varía de un laboratorio a otro y que las dosis equivalentes no son exactas (7) (Tabla I).

En cualquier caso, el sobrecoste del tratamiento con los nuevos opioides retardados (hidromorfona, oxicodona y tapentadol) es mucho mayor que con el empleo de los comercializados hasta ahora, muchos de ellos con genéricos disponibles.

Así las cosas, varios trabajos promovidos por la industria farmacéutica intentan analizar la relación coste-beneficio de la introducción del tapentadol en la práctica clínica (8). En nuestro caso, hemos analizado si la introducción del tapentadol en pacientes con dolor crónico moderado-grave ya tratados con la asociación de otros opioides y pregabalina puede permitir no sólo el cambio de opioide, sino la retirada del neuromodulador (dado el doble efecto descrito del tapentadol), con beneficio clínico para el paciente (mejor control del dolor y menos efectos secundarios) y el coste económico en relación con el tratamiento previo.

\section{PACIENTES Y MÉTODO}

Se trata de un estudio observacional basado en un análisis retrospectivo de los datos (recogidos prospectivamente durante dos meses) proporcionados por pacientes atendidos en una Clínica del Dolor en el ámbito privado que valora unos 150 pacientes semanales pertenecientes a aseguradoras médicas de enfermedades comunes.

Durante este periodo se ha propuesto a pacientes con más de 6 meses de seguimiento con nosotros, en tratamiento con pregabalina asociada a opioides (excepto tapentadol) de más de 3 meses de duración y dolor mal controlado en rango moderado-grave $(\mathrm{EVA}>4)$ rotar a tratamiento con tapentadol sustituyendo primero el opioide y luego la pregabalina. Los criterios de exclusión eran: edad menor de 18 años, dolor oncológico, deterioro cognitivo instaurado e incapacidad para deambulación autónoma.

Este tipo de tratamiento es parte de la práctica habitual de rotación de opioides en pacientes mal controlados, por lo que no se requirió consentimiento específico.

El seguimiento se realizó en tres visitas con intervalos de dos semanas cada una. En la primera se evaluaba el dolor con la escala visual analógica (EVA) y se daba una 
TABLA I. COSTE DIARIO DE TRATAMIENTO PARA DOSIS EQUIPOTENTES DE LOS DIVERSOS OPIOIDES DE USO COMÚN EN ESPAÑA

\begin{tabular}{|c|c|c|c|c|c|c|c|c|c|c|}
\hline Tratamiento & Dosis & Coste* & Dosis & Coste* & Dosis & Coste* & Dosis & Coste* & Dosis & Coste* \\
\hline Morfina, oral & $\begin{array}{c}40 \mathrm{mg} / \\
\mathrm{d}^{1}\end{array}$ & 0,76 & $\begin{array}{c}80 \mathrm{mg} / \\
\mathrm{d}^{2}\end{array}$ & 1,28 & $\begin{array}{c}120 \mathrm{mg} / \\
\mathrm{d}^{3}\end{array}$ & 1,64 & $\begin{array}{c}160 \mathrm{mg} / \\
\mathrm{d}^{4}\end{array}$ & 2,4 & $\begin{array}{c}200 \mathrm{mg} / \\
\mathrm{d}^{5}\end{array}$ & 2,46 \\
\hline Oxicodona, oral & $20 \mathrm{mg} / \mathrm{d}$ & 0,66 & $40 \mathrm{mg} / \mathrm{d}$ & 1,32 & $\begin{array}{c}60 \mathrm{mg} / \\
\mathrm{d}^{6}\end{array}$ & 1,98 & $80 \mathrm{mg} / \mathrm{d}$ & 4,4 & $\begin{array}{c}100 \mathrm{mg} / \\
\mathrm{d}^{7}\end{array}$ & 5,06 \\
\hline $\begin{array}{l}\text { Oxicodona/ } \\
\text { naloxona, oral }\end{array}$ & $\begin{array}{l}20 / 10 \\
\mathrm{mg} / \mathrm{d}\end{array}$ & 1,88 & $\begin{array}{l}40 / 20 \\
\mathrm{mg} / \mathrm{d}\end{array}$ & 3,78 & $\begin{array}{l}60 / 30 \\
\mathrm{mg} / \mathrm{d}^{8}\end{array}$ & 5,66 & $\begin{array}{l}80 / 40 \\
\mathrm{mg} / \mathrm{d}\end{array}$ & 5,74 & $\begin{array}{l}100 / 50 \\
\mathrm{mg} / \mathrm{d}^{9}\end{array}$ & 7,62 \\
\hline Tramadol, oral & $\begin{array}{l}200 \\
\mathrm{mg} / \mathrm{d}\end{array}$ & 0,42 & $\begin{array}{l}400 \\
\mathrm{mg} / \mathrm{d}\end{array}$ & 0,84 & --- & --- & --- & --- & --- & --- \\
\hline $\begin{array}{l}\text { Hidromorfona, } \\
\text { oral }\end{array}$ & $4 \mathrm{mg} / \mathrm{d}$ & 0,9 & $8 \mathrm{mg} / \mathrm{d}$ & 1,8 & $12 \mathrm{mg} /$ & 2,7 & $16 \mathrm{mg} / \mathrm{d}$ & 3,6 & --- & --- \\
\hline $\begin{array}{l}\text { Fentanilo, } \\
\text { transdérmico }\end{array}$ & $12 \mu \mathrm{g} / \mathrm{h}$ & 0,47 & $25 \mu \mathrm{cg} / \mathrm{h}$ & 0,98 & $50 \mu \mathrm{cg} / \mathrm{h}$ & 1,95 & $75 \mu \mathrm{cg} / \mathrm{h}$ & 2,93 & --- & --- \\
\hline $\begin{array}{l}\text { Buprenorfina, } \\
\text { transdérmico }\end{array}$ & --- & --- & $35 \mu \mathrm{cg} / \mathrm{h}$ & 2,02 & $\begin{array}{c}52,5 \\
\mu \mathrm{cg} / \mathrm{h}\end{array}$ & 2,96 & $70 \mu \mathrm{cg} / \mathrm{h}$ & 3,91 & --- & --- \\
\hline Tapentadol, oral & $\begin{array}{c}100 \\
\mathrm{mg} / \mathrm{d}\end{array}$ & 1,46 & $\begin{array}{c}200 \\
\mathrm{mh} / \mathrm{h}\end{array}$ & 2,94 & $\begin{array}{c}300 \\
\mathrm{mg} / \mathrm{d}\end{array}$ & 4,4 & $\begin{array}{c}400 \\
\mathrm{mg} / \mathrm{d}\end{array}$ & 5,12 & $\begin{array}{c}500 \\
\mathrm{mg} / \mathrm{h}\end{array}$ & 5,64 \\
\hline
\end{tabular}

*Coste (precio de venta al público + IVA) en $€$ (euros)

Para morfina oral:

${ }^{1} 4$ comprimidos $10 \mathrm{mg} /$ día

${ }^{2} 1$ comprimido $30 \mathrm{mg}+1$ comprimido $10 \mathrm{mg} 2$ veces al día

32 comprimidos $60 \mathrm{mg}$

${ }^{4} 1$ comprimido $60 \mathrm{mg}+2$ comprimidos $10 \mathrm{mg} 2$ veces al día

${ }^{5} 2$ comprimidos $100 \mathrm{mg} / \mathrm{dia}$

Para oxicodona oral:

${ }^{6} 1$ comprimido $400 \mathrm{mg}+1$ comprimido $10 \mathrm{mg} 2$ veces al día

${ }^{7} 1$ comprimido $40 \mathrm{mg}+1$ comprimido $10 \mathrm{mg} 2$ veces al día

Para oxicodona/naloxona oral:

${ }^{8} 1$ comprimido 20/10 mg +1 comprimido 10/5 mg 2 veces al día

${ }^{7} 1$ comprimido 40/20 mg +1 comprimido 10/5 mg 2 veces al día

Para hidromorfona oral:

${ }^{10} 1$ comprimido $4 \mathrm{mg}+1$ comprimido $8 \mathrm{mg}$

(Fuente: www.msssi.es. Nomenclátor de facturación. Precios a 1 de noviembre de 2012).

pauta adaptada a cada caso para retirar el opioide e introducir tapentadol basado en las equivalencias de dosis. En caso de que percibiera aparición o aumento de los efectos secundarios característicos (náuseas, vómitos, estreñimiento, mareo...) se le recomendaba volver al tratamiento previo. En la segunda visita, a los pacientes que no abandonaron el nuevo tratamiento se les proporcionó una nueva pauta para retirar progresivamente la pregabalina y otra para subir la dosis de tapentadol a razón de $50 \mathrm{mg}$ cada 3 días hasta conseguir un adecuado control del dolor sin secundarismos. En la última visita se apuntaba la dosis final alcanzada por cada paciente y se reevaluaba el dolor con la EVA.

Basándonos en los precios de venta al público (PVP + IVA) se calculó el coste de tratamiento en cada caso antes y después del cambio, así como el coste global.
Los datos se expresan como media \pm E.S.M. Para el análisis estadístico de resultados de la EVA y coste con uno y otro tratamiento se empleó la t de Student para datos pareados. Se considera significativa una $\mathrm{p}<0,05$.

\section{RESULTADOS}

Se reclutaron 21 pacientes con edad media de 65,93 años (15 mujeres y 6 varones). Seguían tratamiento previo con pregabalina asociada a diversos opioides: tramadol 4, hidromorfona 2 , oxicodona u oxicodona/naloxona 8 , fentanilo transdérmico 6 y buprenorfina transdérmica 1 (Tabla II).

El diagnóstico más común era dorsalgia/lumbagia crónica con $(\mathrm{n}=8)$ o sin radiculopatía $(\mathrm{n}=10)$. En ambos 
TABLA II. DOSIS MEDIA DE LOS DISTINTOS OPIOIDES ADMINISTRADOS AL INICIAR LA ROTACIÓN

\begin{tabular}{lc}
\hline \multicolumn{1}{c}{ Fármaco $(\mathrm{n})$} & Dosis media \\
\hline Tramadol (4) & $268,13 \mathrm{mg}$ \\
Hidromorfona (2) & $16 \mathrm{mg}$ \\
Oxicodona (8) & $38,42 \mathrm{mg}$ \\
Fentanilo (6) & $50 \mu \mathrm{g} / \mathrm{h}$ \\
Burprenorfina (1) & $52,5 \mu \mathrm{g} / \mathrm{h}$ \\
\hline
\end{tabular}

grupos el $50 \%$ tras cirugía fallida de columna. Tres casos presentaban poliartrosis grave como diagnóstico principal.

Tras la primera visita cuatro pacientes abandonaron el tratamiento para volver a su pauta habitual (Fig. 1). En todos los casos fue por aumento de los efectos secundarios al introducir el tapentadol, fundamentalmente sequedad de boca y mareo/inestabilidad.

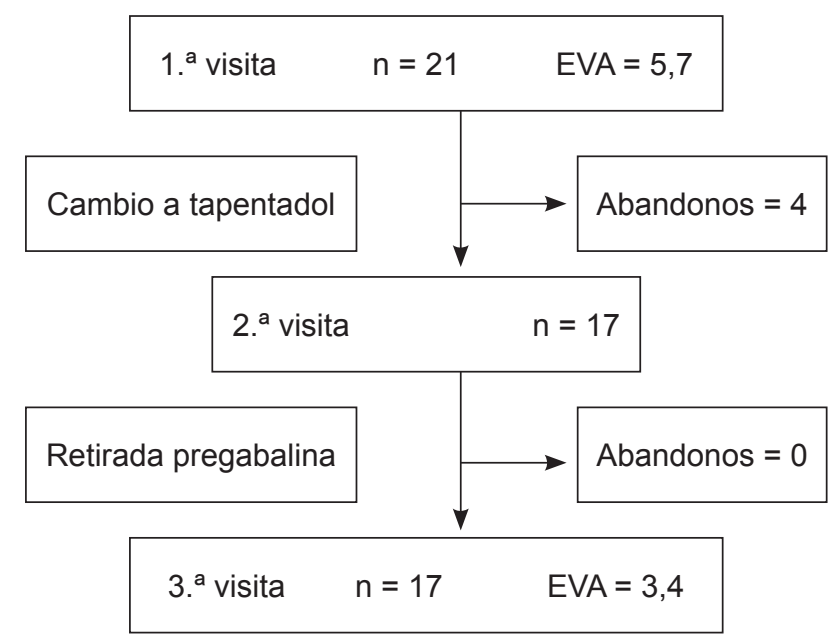

Fig. 1. Diagrama de flujo de pacientes en las tres visitas.

Tras la segunda visita ningún paciente abandonó, al tener que retirar la pregabalina, y en todos los casos se pudo subir la dosis de tapentadol.

La dosis media final de tapentadol en los 17 pacientes valorados en la última visita fue de $335,71 \pm 37 \mathrm{mg} /$ día, con un coste de $3,78 \pm 0,71 € /$ día, frente a $4,57 \pm 0,84 € /$ día $(p<0,05)$ de coste medio del tratamiento previo. Considerados caso a caso, en 13 pacientes la introducción de tapentadol resultaba más económica y en 4 casos más cara, aunque en estos el sobrecoste suponía $0,73 €$ /día por término medio.

En cuanto a la eficacia clínica, el control del dolor en los pacientes con tapentadol supuso una mejoría de un $40,35 \%(p<0,001)$ respecto a su tratamiento previo.

\section{DISCUSIÓN}

La comercialización de nuevos fármacos opioides en los últimos años, concretamente oxicodona, hidromorfona y tapentadol, ha supuesto una pequeña revolución en el manejo del dolor moderado-grave, tanto agudo como crónico. No se trata sólo de nuevas moléculas (algunas, como la hidromorfona, llevan muchos años comercializadas en otros países), sino de nuevas presentaciones galénicas que permiten: a) administrar dosis extremadamente bajas bioequivalentes con dosis moderadas de codeína y bajas de tramadol; b) preparados de acción inmediata para titulación de dosis, rescate y dolor irruptivo; c) preparados retard de $12 \mathrm{~h}$ y $24 \mathrm{~h}$; d) formulaciones líquidas para casos de dificultades con la deglución; y e) presentaciones inyectables.

No todos los opioides son iguales y sus características farmacocinéticas y famacodinámicas marcan la diferencia. La introducción de estas moléculas ha permitido mejorar notablemente el control analgésico de pacientes con dolor crónico que no se podían controlar con las moléculas existentes, bien por falta de eficacia o por intolerancia a los secundarismos. En nuestra Unidad del Dolor el número de implantes espinales para administración de mórficos por vía epidural o intratecal (catéteres permanentes, reservorios epidurales y bombas intradurales) se ha reducido más de un $70 \%$ en los últimos 5 años, coincidiendo con la aparición en el mercado español de estos opioides.

El problema del sobrecoste del tratamiento al introducir estos nuevos opioides y la duda permanente entre la relación coste-beneficio afecta tanto al ámbito sanitario público como al privado, especialmente en los tiempos de crisis y de intento de control del gasto farmacéutico que vivimos. En nuestro centro atendemos muchos pacientes de los colectivos de funcionarios del estado (MUFACE, MUGEJU, ISFAS) adscritos a seguros médicos privados, que cargan con una parte del coste de la medicación prescrita. Esto nos obliga también a analizar seriamente si los tratamientos que prescribimos están justificados en relación con el beneficio que proporcionan al paciente y el coste comparado con otros tratamientos.

El tapentadol, al ser un fármaco con acción dual (analgésico opioide + neuromodulador), permite en la mayoría de los casos retirar el antiepiléptico pregabalina asociado a otros opioides convencionales y, en nuestra experiencia, de una forma coste-efectiva: mejorando el control del dolor, disminuyendo los efectos secundarios y la polifarmacia y resultando incluso algo más barato que el tratamiento previo en la mayoría de los casos.

Existe algún estudio en modelo animal que plantea que la asociación de pregabalina con tapentadol puede resultar interesante en el tratamiento del dolor neuropático al asociar dos efectos neuromoduladores sinérgicos (antagonismo del $\mathrm{Ca}^{2+}$ e inhibición de recaptación de noradrenalina) (9). Sin embargo, los resultados preliminares de un ensayo clínico en fase III parece mostrar que en humanos la 
mejoría clínica no es significativa, mientras que los efectos secundarios sí que se multiplican de forma estadísticamente muy significativa (Ralf Baron, Comunicación personal, 14 ${ }^{\text {th }}$ World Congress on Pain (IASP), Milán, agosto 2012).

Nuestra muestra parece apuntar en sentido contrario: tapentadol aislado es más eficaz que la combinación de otros opioides asociados a pregabalina y mejora la tolerancia. Sin embargo, un $19 \%$ de los pacientes de nuestra serie prefirieron mantener el tratamiento previo por mala tolerancia al tapentadol, lo que permite recordar que las variables farmacocinéticas, farmacodinámicas y farmacogenómicas tienen mucho que decir a la hora de valorar la distinta respuesta a cada opioide por cada paciente.

Se trata de una muestra pequeña y serían necesarios estudios multicéntricos y con un diseño más complejo para poder llegar a conclusiones definitivas. Pero sí parece razonable afirmar, por lo visto en este estudio, que la rotación a tapentadol en pacientes con dolor crónico moderado-grave no bien controlado en tratamiento con otros opioides + pregabalina es una opción que puede mejorar el control del dolor, disminuir los secundarismos y reducir la polifarmacia, sin suponer un aumento del coste e incluso reduciéndolo.

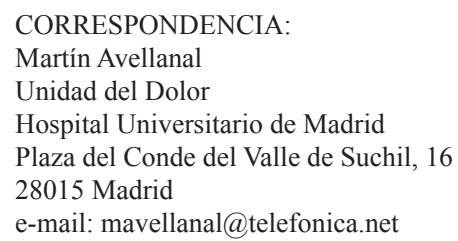

\section{BIBLIOGRAFÍA}

1. Kress HG. Tapentadol and its two mechanisms of action: Is there a new pharmacological class of centrally-acting analgesics on the horizon. Eur J Pain 2010;14:781-3.

2. Afilalo M, Etropolski MS, Kuperwasser B, Kelly K, Okamoto A, Van Hove I, et al. Efficacy and safety of tapentadol extended release compared with oxycodone controlled release for the management of moderate to severe chronic pain related to osteoarthritis of the knee: A randomized, double blind, placebo- and active-controlled phase III study. Clin Drug Investig 2010;30:489-505.

3. Tzschentke TM, Christoph T, Kogel B, Schiene K, Hennies HH, Englberger W, et al. (-)-(1R,2R)-3-(3-dimethylamino1-ethyl-2-methyl-propyl)-phenol hydrochloride (tapentadol $\mathrm{HCl}$ ): A novel mu-opioid receptor agonist/norepinephrine reuptake inhibitor with broad-spectrum analgesic properties. J Pharmacol Exp Ther 2007;323:265-76.

4. Millan MJ. Descending control of pain. Prog Neurobiol 2002;66:355-474.

5. Frampton JE. Tapentadol inmediate release. A review of its use in the treatment of moderate to severe acute pain. Drugs 2010;70:1719-43.

6. Merker M, Dinges G, Koch T, Kranke P, Morin AM. Unerwünschte Nebenwirkungen von Tapentadol in vergleich zu Oxycodon. Eine Metaanalyse randomisierter, kontrollierter Vergleichsstudien. Schmerz 2012;26:16-26.

7. Torres LM. Tapentadol Retard en el dolor crónico intenso. Rev Soc Esp Dol 2011;18:283-90.

8. Obradovic M, Ikenberg R, Hertel N, Antoñanzas F, Gálvez $\mathrm{R}$, Liedgens H. Cost-effectiveness of tapentadol in severe chronic pain in Spain: A cost analysis of data from RCTs. Clin Ther 2012;34:926-43.

9. Christoph T, De Vry J, Schiene K, Tallarida RJ, Tzschentke TM. Synergistic antihypersensitive effects of pregabalin and tapentadol in a rat model of neuropathic pain. Eur J Pharmacol 2011;666:72-9. 